\title{
Visibilidad de las revistas electrónicas latinoamericanas en las bases de datos internacionales
}

Latin American electronic journals: its visibility through databases

\author{
María Guadalupe Trinidad Argüello Mendoza (1) y Sergio MÁRquez RANGel (2) \\ Dirección General de Bibliotecas, Universidad Nacional Autónoma de México, C/ Circuito Exterior, s/n, \\ Edificio Biblioteca central, Ciudad Universitaria, 04510 México, D. F. (México) (1) smar- \\ quez@dgb.unam.mx(2)gmendoza@dgb.unam.mx.
}

\begin{abstract}
Resumen
Análisis del impacto de las revistas en formato electrónico que son de países latinoamericanos y que son incluidas en las bases de datos internacionales, para medir la visibilidad de estas publicaciones en la literatura internacional. Se trabajo con las revistas latinoamericanas seleccionadas, analizadas y resguardas en la Hemeroteca Latinoamericana de la Dirección General de Bibliotecas de la Universidad Nacional Autónoma de México (UNAM) y que están en formato electrónico tanto para su consulta como para su almacenamiento y que son incluidas en los índices internacionales producidos por esta universidad: CLASE y PERIODICA. Se buscaron estos títulos dentro de las bases de datos más importantes de la disciplina para detectar cuantos títulos son incluidos, su cobertura y estatus. Los datos emanados se analizaron, tabularon e interpretaron para emitir un diagnostico sobre el comportamiento de estas publicaciones a nivel internacional. Finalmente se buscaron las revistas en el Journal Citation Report para conciliar los títulos localizados con su factor de impacto. Los datos obtenidos de las diferentes bases de datos analizadas demuestran que las revistas latinoamericanas no son incluidas en ellas, que los países con mayor crecimiento económico son los que sobresalen en las estadísticas y que las áreas temáticas que resaltan en este ejercicio son las biomédicas.
\end{abstract}

Palabras clave: Bases de datos. América Latina. Revistas electrónicas. Factor de impacto.

\section{Introducción}

Las revistas Latinoamericanas siempre han sufrido el problema de la difusión, aunado a la problemática de la región, donde el impulso a la actividad científica se ve disminuido, los problemas sociales son comunes y las condiciones económicas siempre son inestables.

Ello impide que las revistas de esta región puedan cumplir los estándares de inclusión en las bases de datos internacionales. Por lo tanto, no

\begin{abstract}
An assessment of the impact of Latin American electronic journals included in international databases is offered, with the aim of evaluating the performance of these journals in the international literature. The Latin American journals selected are analyzed and preserved in the Latin American Journal Collection (Hemeroteca Latinoamericana) of the General Libraries Directorate of the National University of Mexico (UNAM); are available on line and included in two databases, namely PERIODICA and CLASE, were used for this work. The journal titles were searched in the most important international databases of each discipline with the aim of detect their presence in this databases their coverage and actual status. The obtained data were analyzed and interpreted. A diagnosis on the performance of these journals at an international level was realized. Finally, these journals were searched in the Journal Citation Report, just to know their impact factor. Results showed that the Latin American journals are not included in the most important international databases, and also that the most developed countries have a higher presence in these databases, and that the most outstanding discipline is biomedicine.
\end{abstract}

Keywords: Latin America. Scientific literature. Electronic Journals. Database selection. Impact factor

hay una penetración de la ciencia latinoamericana a nivel mundial; salvo los científicos que publican en las revistas de alto factor de impacto o que salen de sus países para poder tener mejores perspectivas en el crecimiento profesional. Es así como se pretende analizar el comportamiento este tipo de publicaciones dentro de las bases de datos internacionales.

Se decidió trabajar con las revistas que son adquiridas, organizadas y resguardadas en la Hemeroteca Latinoamericana de la Dirección 
General de Bibliotecas de la UNAM, las cuales son empleadas para el desarrollo de las bases de datos de citas latinoamericanas: CLASE (Base de Datos Bibliográfica en Ciencias Sociales y Humanidades), PERIODICA (Base de Datos Bibliográfica en Ciencias y Tecnología) y ASFA (Aquatic Sciences and Fisheries Abstracts).

Esta Hemeroteca es el resultado de la reunión celebrada en el Centro Médico Nacional en 1975, en el que se percibió la necesidad de contar con todo el material publicado sobre el tema de las ciencias sociales en Latinoamérica para elaborar el índice CLASE. Éste se empezó a publicar en 1975. Así surgió la idea de contar con una hemeroteca y en 1976 se empezó a formar ésta de la que se hace mención por primera vez en el informe de la UNAM de ese año (Dirección de Asuntos Administrativos, 1977).

Por su parte, la base de datos PERIODICA se empezó a publicar desde el año 1978. De estas mismas publicaciones se hace un concentrado para la base de datos ASFA.

ASFA se crea en 1980 gracias a un convenio entre la UNAM y la Organización de las Naciones Unidas para la Agricultura y la Alimentación (FAO) y se aprovechó la infraestructura que tenía el entonces Centro de Información Científica y Humanística $(\mathrm{CICH})$ para poder analizar la información Latinoamericana y contribuir con el desarrollo de la base.

Es así como se crea en el $\mathrm{CICH}$ una oficina para el Centro Regional ASFIS (Aquatic Sciences and Fisheries Information System) para la América Latina y el Caribe. El sistema ASFIS tiene como uno de sus propósitos principales el elaborar la base de datos ASFA (Aquatic Sciences and Fisheries Abstracts). Así, el $\mathrm{ClCH}$, en su calidad de miembro de ASFA, se encargó, a partir de esa fecha y hasta la actualidad, de recopilar, analizar y sistematizar toda la información sobre ciencias acuáticas y pesca que se genera en Latinoamérica.

También en esa época se producían otras dos bases de datos: BLAT y CIN, las cuales estaban especializadas en Literatura Latinoamericana y Ciencias de la información respectivamente; pero se dejaron de trabajar en 1994 por la duplicidad de trabajos tanto con CLASE y PERIODICA, como con otras que se desarrollaban al interior de UNAM (Tapia, 2008).

Al inicio, la hemeroteca del $\mathrm{CICH}$ recibió 450 títulos de publicaciones periódicas (51\% por suscripción y el $49 \%$ por donación). De ellas, el $56 \%$ pertenece a servicios de índices o resúmenes en diversos campos de las ciencias y de las humanidades y el resto lo integran publicaciones periódicas primarias (Dirección de Asuntos Administrativos, 1976).

Con el surgimiento de las nuevas herramientas para trabajar con publicaciones en formato digital y el advenimiento propiamente dicho de las revistas electrónicas, los países de la región iniciaron sus trabajos para digitalizar sus revistas. A la fecha este esfuerzo ha sido mínimo, pero consolidado.

La Hemeroteca Latinoamericana actualmente cuenta con 3500 títulos y 86,500 fascículos, y cuenta con 460 títulos que se encuentran en forma electrónica algunos de ellos se encuentran almacenados en CD-ROM y otros solo cuentan con el enlace a la paginas del editor 0 el portal de la revista. (Luna, 2004)

Si comparamos estos datos con los proporcionados por el Directorio Internacional de Publicaciones Periódicas Ulrich's se puede apreciar que la HELA solo cuenta con el $27 \%$ del total de las publicaciones vigentes en la región, ya que su acervo esta constituido por 3500 títulos y en la región se publican más de 12 mil títulos.

De acuerdo con Ulrich's los países con mayor numero de publicaciones son Brasil con 25\%, Argentina y México con el $14 \%$, y los demás países de la región representan el $20 \%$ del total de los títulos.

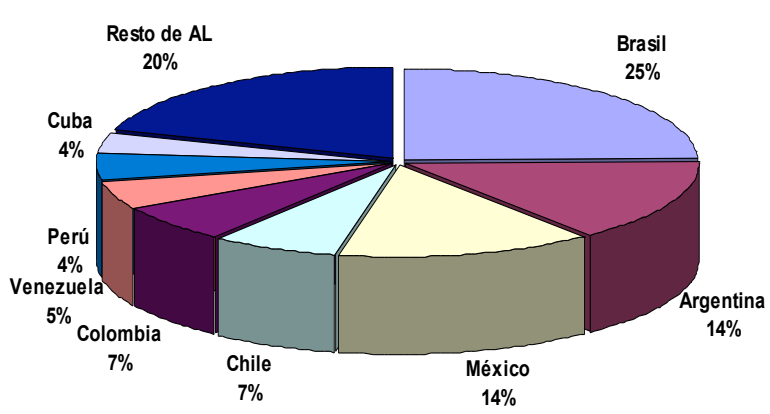

Gráfico 1. Revistas Latinoamericanas por país. [Fuente: Ulrich's]

Si se comparan los datos de Ulrich's con los que ofrece Latindex encontramos una gran diferencia entre el número de títulos ya que en su directorio nos indica el registro de 16 mil títulos pero incluyen a España y Portugal.

Si se observa con detalle la información proporcionada por Latindex, se aprecia que en algunos países de la región disminuyen sus publicaciones o no son indizadas. En el caso de Brasil se incrementa en $11 \%$, en el de México en $22 \%$, y Argentina sube hasta el $30 \%$. 
En este caso se utilizan criterios de inclusión en los dos directorios totalmente diferentes, lo que trae consigo que no se pueda apreciar la realidad de los títulos que son publicados en la región. Ya que, por un lado, los criterios de UIrich's son básicamente comerciales; y, por el otro, no incluye realmente todo el mercado latinoamericano.

Por su parte Latindex -con sus criterios académicos - elimina muchos de los títulos de países pequeños; y, en el caso de los países grandes de la región, incluye otra cantidad impresionante de títulos que difieren.

Al comparar los resultados de Ulrich's y Latindex con los títulos que están incluidos en HELA, se observa que baja considerablemente la cantidad de títulos y que éstos representan tan solo el $27 \%$ de las publicaciones. Si se compara con las revistas electrónicas, el número disminuye hasta llegar al $6 \%$.

Los países incluidos permanecen, pero cambian de posición. Hay que recordar que los títulos de la Hemeroteca Latinoamericana de la UNAM son evaluados con criterios más severos -a diferencia de Latindex. Además, la inclusión de estos títulos no es tan fácil como se pensaría, por eso solo se trabajan 3500 títulos en formato impreso, de los cuales 421 están en formato electrónico.

Los criterios de CLASE y PERIODICA están divididos en: normalización editorial, gestión y visibilidad y los relacionados con los contenidos con fines de indización (Alonso, 2003).

\begin{tabular}{lc}
\hline País & Títulos \\
\hline México & 175 \\
Brasil & 103 \\
Colombia & 46 \\
Chile & 43 \\
Argentina & 22 \\
Venezuela & 17 \\
Costa Rica & 7 \\
Cuba & 4 \\
Ecuador & 3 \\
Perú & 3 \\
\hline
\end{tabular}

Tabla I. Títulos de revistas electrónicas de HELA por país.

\section{Materiales y métodos}

Se recopilo un listado de 421 títulos con los campos país, frecuencia, acervos, editor, disciplina, editor responsable, base a la que pertenece y el año que ingreso a la hemeroteca. Se logro integrar las publicaciones que se encuen- tran en texto completo y a su vez están en formato impreso.

Se dividió el listado por área temática: físicomatemáticas e ingeniería, ciencias biológicas y de la salud, ciencias sociales, y artes y humanidades.

Cada uno de estos listados se buscó dentro de las dos bases principales de las áreas antes mencionadas, siendo estas respectivamente para cada área: Inspec y MathSciNet, Biological Abstracts y PubMed, Sociological Abstracts y Pscylnfo, y, finalmente, Art Full Text y Humanities Index.

Se han considerado estas bases de datos bajo los siguientes criterios:

- Están disponibles en las colecciones digitales de la UNAM.

- Cuentan con el mayor número de registros bibliográficos en su especialidad.

- Cuentan con el mayor número de revistas especializadas en las áreas que se investiga.

- Tienen un uso alto de acuerdo con las estadísticas obtenidas de los sistemas de los propios proveedores.

Además, estas bases de datos no son del mismo editor o proveedor; y, por tanto, las plataformas de búsqueda son diferentes y permiten obtener diferentes puntos de vista.

De estas búsquedas se extrajeron los datos de cobertura, temática y total de registros, para poder tener los indicadores mínimos que permitieran evaluar la forma en que se han incluido.

Finalmente se hizo la búsqueda de estos mismos títulos en las bases de datos Journal Citation Report Science Edition y Journal Citation Report Social Science Edition del Institute for Scientific Information.

Ello para conocer el número de revistas que han sido sometidas a estándares más rigurosos y que han podido mantenerse en estas bases de excelencia, obteniendo además de los indicadores ya mencionados el factor de impacto.

\section{Resultados}

Los resultados obtenidos son realmente interesantes y confirman muchas teorías que se tenían sobre las colecciones pero que hasta ahora no se podían demostrar con los datos obtenidos. 
3.1. Análisis de los títulos latinoamericanos en Ciencias y Tecnología

Se trabajo con 209 títulos que están incluidos en la base de datos PERIODICA, de los cuales solo se localizó un total de 97 títulos en las cuatro bases que cubren esta temática. En la tabla 2 se aprecian los porcentajes obtenidos. El $46 \%$ de los títulos fueron localizados por lo menos en una base de datos. De estos 97 , el $67 \%$ son títulos únicos y el resto se duplican en más de una base.

\begin{tabular}{lcc}
\hline Concepto & Títulos & $\%$ \\
\hline Total de revistas & 209 & \\
Revistas indexadas & 97 & 46 \\
Títulos únicos & 65 & 67 \\
Títulos duplicados & 32 & 33 \\
\hline
\end{tabular}

Tabla II. Títulos de Periodica localizados en las bases de datos

Se puede observar que las revistas en las áreas de Ciencias Biológicas y de la Salud son las que predominan respecto a la cantidad de títulos latinoamericanos incluidos; ya que la base de datos que mas títulos reporta es Biological Abstracts. Sin embargo, ésta duplica 13 títulos con PubMed, por su parte PubMed es la segunda base que cuenta con la inclusión de 40 títulos.

\begin{tabular}{lc}
\hline Base de Datos & Total \\
\hline Biological Abstracts & 48 \\
PubMed & 40 \\
MathSciNet & 5 \\
Inspec & 4 \\
\hline
\end{tabular}

Tabla III. Títulos localizados en las bases de datos de Ciencias Biológicas y Físico-Matemáticas e Ingenierías

En el caso de Biological Abstracts se puede apreciar que, de los 48 títulos, 9 son cesados; es decir, ya fueron retirados del análisis de las bases de datos. Los países que cuentan con publicaciones son: Brasil con 20 títulos y México con 16; el resto de los países tienen números muy pequeños, como se aprecia en la Tabla 5.

\begin{tabular}{lc}
\hline Títulos & 48 \\
Únicos & 34 \\
Retirados & 6 \\
Duplicados & 14 \\
Retirados duplicados & 3 \\
\hline
\end{tabular}

Tabla IV. Estatus de los títulos localizados en el Biological Abstracts

\begin{tabular}{lcc}
\hline País & Títulos & Retirados \\
\hline Brasil & 20 & 3 \\
México & 16 & \\
Argentina & 5 & 2 \\
Chile & 5 & \\
Colombia & 1 & 1 \\
Costa Rica & 1 & \\
\hline Total & 48 & 6 \\
\hline
\end{tabular}

Tabla V. Países con títulos incluidos en el Biological Abstracts

En PubMed se observa que son 40 títulos los que están incluidos en la base de datos y se repiten los trece títulos duplicados con Biological Abstracts.

\begin{tabular}{ll}
\hline Títulos & 40 \\
Únicos & 27 \\
Retirados & 14 \\
Duplicados & 13 \\
\hline
\end{tabular}

Tabla VI. Estatus de los títulos localizados en PubMed

Por su parte los países que representan estos 40 títulos de revistas electrónicas latinoamericanas son encabezados por Brasil y México. Los países con menos de cuatro títulos tienen un problema adicional, y es que sus títulos en formato electrónico para las Ciencias Biológicas y de Salud en el PubMed están siendo retirados.

\begin{tabular}{lcc}
\hline País & Títulos & Retirados \\
\hline Brasil & 18 & 4 \\
México & 9 & 2 \\
Chile & 4 & 2 \\
Colombia & 2 & 2 \\
Costa Rica & 2 & 1 \\
Cuba & 2 & 2 \\
Venezuela & 2 & 1 \\
Argentina & 1 & \\
\hline Total & 40 & 14 \\
\hline
\end{tabular}

Tabla VII. Países con títulos incluidos en PubMed

Desde la otra perspectiva de las ciencias, se puede apreciar que los número son totalmente diferentes. En las bases de datos especializadas de los temas de Físico-Matemáticas e Ingenierías, los títulos latinoamericanos casi no son incluidos, lo que indica que la representatividad de la región es casi nula; y eso a pesar ade que países como Brasil y México tienen una producción muy grande en este tema, documentada en revistas las internacionales de la especialidad. 


\begin{tabular}{ll}
\hline Títulos & 5 \\
Únicos & 3 \\
Retirados & 1 \\
Duplicados & 2 \\
\hline
\end{tabular}

Tabla VIII. Estatus de los títulos localizados en MathSciNet

Al analizando MathSciNet solo se localizaron cinco títulos de revistas electrónicas latinoamericanas y en Inspec se obtuvieron tan solo cuatro; pero, de estos títulos, dos son duplicados entre ambas bases, por lo que el número disminuye aun más.

Por su parte los países que representan estos cinco títulos en esta ocasión están lideriados por México; ya que comparte la misma cantidad con Brasil, pero uno de sus títulos esta retirado de la base de datos, como se aprecia en la tabla 9.

\begin{tabular}{lcc}
\hline País & Títulos & Retirados \\
\hline México & 2 & \\
Brasil & 2 & 1 \\
Colombia & 1 & \\
\hline Total & 5 & 1 \\
\hline
\end{tabular}

Tabla IX. Países con títulos incluidos en MathScinet

Para el análisis de los títulos localizados en la base de datos Inspect se detectaron cuatro títulos, de los cuales dos ya se reportaron como duplicados en la base MathSciNet y un tercero, por extrañas razones, se duplica con la base Biological Abstracts.

\begin{tabular}{ll}
\hline Títulos & 4 \\
Únicos & 1 \\
Retirados & 1 \\
Duplicados & 3 \\
\hline
\end{tabular}

Tabla X. Estatus de los títulos localizados en Inspec

\begin{tabular}{lcc}
\hline País & Títulos & Retirados \\
\hline México & 2 & \\
Brasil & 1 & \\
Colombia & 1 & 1 \\
Total & 4 & 1 \\
\hline
\end{tabular}

Tabla XI. Países con títulos incluidos en Inspec

Estos cuatro títulos de la colección de la Hemeroteca Latinoamericana están representados por México con dos títulos, Brasil y Colombia con un título, aunque el título de este último país ya fue retirado de la colección.
3.2. Análisis de los títulos indexados en Ciencias Sociales y Humanidades

En el caso de las de las áreas de Ciencias Sociales y Humanidades es sabido que cuentan con publicaciones con un contenido temático muy rico, ya que es la faceta que mejor desarrollan las universidades de la región. Sin embargo, al igual que las publicaciones del área FísicoMatemática e Ingenierías, éstas no son muy visibles en las grandes bases de datos internacionales. De la búsqueda realizada se puede observar que - de las 212 revistas que cubren los temas y que están indexadas en la base de datos CLASE- solo se localizaron 35 títulos en dos bases internacionales.

\begin{tabular}{lc}
\hline Total de revistas & 212 \\
\hline Revistas indexadas & 35 \\
Títulos únicos & 34 \\
Títulos duplicados & 1 \\
\hline
\end{tabular}

Tabla XII. Títulos localizados en las bases de datos de Ciencias Sociales y Artes y Humanidades

Las bases que reportaron registros sobre estas revistas latinoamericanas en formato electrónico fueron Sociological Abstracts y Psyclnfo. Las bases Humanities y Art Abstracts no incluyen ninguno de los 212 títulos.

\begin{tabular}{lc}
\hline Base de Datos & Total \\
\hline Sociological Abstracts & 28 \\
Psyclnfo & 7 \\
\hline
\end{tabular}

Tabla XIII. Títulos de CLASE localizados en las bases de datos

En la tabla 14 se aprecia el estatus de los títulos localizados en la base de datos Sociological Abstracts. En esta base se detectaron 28 títulos, de los cuales todos tienen una cobertura vigente y solo uno de ellos se duplica con Psyclnfo.

\begin{tabular}{lc}
\hline Títulos & 28 \\
Únicos & 27 \\
Retirados & 2 \\
Duplicados & 1 \\
\hline
\end{tabular}

Tabla XIV. Estatus de los títulos localizados en Sociological Abstracts

Los países que representan estos 28 títulos localizados son encabezados por México con 14 títulos y seguido por Brasil con 9; los demás países Latinoamericanos cuentan con una cantidad mínima. 


\begin{tabular}{lcc}
\hline País & Títulos & Retirados \\
\hline México & 14 & 1 \\
Brasil & 9 & \\
Venezuela & 2 & \\
Argentina & 1 & \\
Colombia & 1 & \\
Ecuador & 1 & 1 \\
Total & 28 & 2 \\
\hline
\end{tabular}

Tabla XV. Países con títulos incluidos en Sociological Abstracts

Psyclnfo - al igual que todas las bases de datos ya mencionadas - incluye una cantidad importante de revistas latinoamericanas, pero son en formato impreso y las que están en formato electrónico tan solo son siete, lo que puede sugerir que este tipo de recurso no es bien aceptado por la bases de datos internacionales.

\begin{tabular}{ll}
\hline Títulos & 7 \\
\hline Únicos & 6 \\
Retirados & 1 \\
Duplicados & 1 \\
\hline
\end{tabular}

Tabla XVI. Estatus de los títulos localizados en Psyclnfo

Los países que representan estos siete títulos son dos: Brasil con 6 y México que, aunque cuenta con un título, este ya fue retirado del análisis de la base de datos.

\begin{tabular}{lcc}
\hline País & Títulos & Retirados \\
\hline Brasil & 6 & \\
México & 1 & 1 \\
Total & 7 & 1 \\
\hline
\end{tabular}

Tabla XVII. Países con títulos incluidos en Psyclnfo

Lo que arroja realmente este estudio podrían ser buenas noticias o todo lo contrario. De una lista de 421 títulos, solo se localizaron 97 de las áreas de ciencias y 35 de las ciencias sociales, lo que representa en suma 132 títulos, es decir, el $31 \%$ de la colección de revistas incluidas en la Hemeroteca Latinoamericana de la UNAM.

Las áreas de ciencias - aun para el caso de Latinoamérica- siguen siendo las que superan en número a las ciencias sociales y con un $40 \%$, pero las revistas en Ciencias Biológicas y de la Salud cuentan con una gran cantidad de títulos retirados de las bases de datos.

Por su parte las bases de datos de Ciencias Sociales y Humanidades representan el menor porcentaje en títulos retirados de sus conteni- dos, ya que si se comparan los 3 títulos retirados de 35 encontrados, contra los 20 retirados de 97 en Ciencias y Tecnología; podemos deducir que las Ciencias Sociales tienen más permanencia.

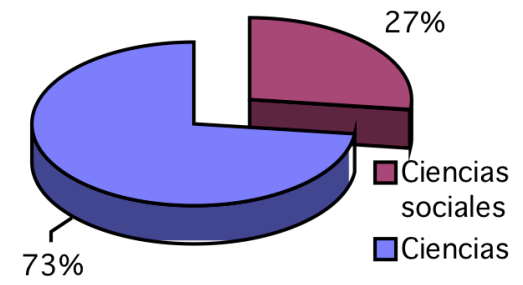

Grafico 2. Distribución de los títulos localizados en las bases da datos por área

También se puede destacar que los países que sobresalen en ciencias y tecnología son Brasil con 41 títulos -que representan el $43 \%$ del total-y México con 29 títulos - con el $30 \%$. También figuran Chile - $9 \%$-, Argentina $6 \%$-, Colombia - 5\%-, Costa Rica - 3\%-y Venezuela y Cuba - con el $2 \%$ cada uno.

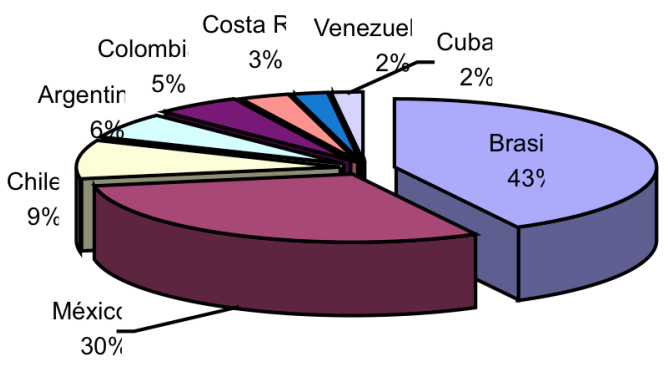

Grafico 3. Porcentaje de títulos localizados en las bases da datos por país en ciencias

De igual forma para las ciencias sociales y humanidades, el país que domina, aunque por poco, es Brasil con el $43 \%$ de los títulos identificados. México le sigue muy de cerca con el $42 \%$, pero la diferencia es que el grueso de los títulos de México se localizó en una sola base de datos, el Sociological Abstracts.

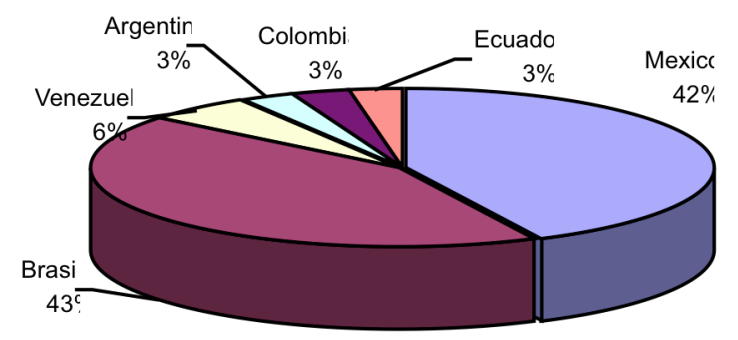

Grafico 4. Porcentaje de títulos localizados en las bases da datos por país en ciencias sociales 


\subsection{Factor de impacto}

De los 421 títulos de revistas latinoamericanas en formato electrónico, se detectó una cantidad muy representativa de títulos incluidos en el Journal Citation Report Science Edition. Se encontraron 21 de los 97 títulos del tema, de los cuales 19 pertenecen a las Ciencias Biológicas y de la Salud, y 3 títulos al tema de FísicoMatemáticas e Ingenierías.

En ellos se reitera el dominio de los títulos ubicados en el área de Ciencias Biológicas y de la Salud, pero el vuelco que da es que el factor de impacto mas alto detectado pertenece a las Ciencias Físico-Matemáticas e Ingenierías y, concretamente, a una publicación mexicana.

\begin{tabular}{lc}
\hline Título & $F I$ \\
\hline Revista Mexicana de Astronomía y & 2.094 \\
Astronomía & \\
Memorias do Instituto “Oswaldo Cruz" & 1.208 \\
Biological Research & 1.177 \\
Brazilian Journal of Medical \& & 1.075 \\
Biological Research & \\
Electronic Journal Biotechnology & 0.767 \\
Revista Mexicana Ciencias & 0.682 \\
Geológicas & \\
Revista Chilena de Historia Natural & 0.646 \\
Genetics and Molecular Biology & 0.574 \\
Brazilian Journal Physics & 0.494 \\
Ciencias Marinas & 0.439 \\
Revista Medica de Chile & 0.405 \\
Arquivos de Neuro-Psiquiatría & 0.400 \\
Revista Brasileña de Zootecnia & 0.385 \\
Atmósfera & 0.344 \\
Revista Mexicana de Física & 0.265 \\
Brazilian Archives of Biology and & 0.225 \\
Technology & \\
Brazilian Journal of Microbiology & 0.213 \\
Archivos de Medicina Veterinaria & 0.130 \\
Acta Bioquímica Clínica & 0.125 \\
Latinoamericana & \\
Agrociencia & \\
Arquivo Brasileiro de Medicina & \\
Veterianria y Zootecnia & 0.1117 \\
\hline
\end{tabular}

Tabla 18. Revistas localizadas en el JCRS

Los países a los que pertenecen estas revistas electrónicas incluidas en el Journal Citation Reports Science Edition (JCRS) son Brasil con 9 títulos, México con 6 , Chile con 5 y Argentina con uno. Vemos que el predominio corresponde a los países mas desarrollados.

Por otro lado, y reiterando el problema de las revistas en Ciencias Sociales y Humanidades, solo se detectaron cuatro títulos de las revistas electrónicas en el Journal Citation Report Social Science Edition (JCRSS). Además, los números de factor son realmente bajos como podemos apreciar en la tabla 19.

\begin{tabular}{lc}
\hline Publicaciones & $F I$ \\
\hline Salud Pública de México & 0.536 \\
Revista latinoamericana de psicología & 0.400 \\
Dados & 0.102 \\
Eure (Santiago) & 0.057 \\
\hline
\end{tabular}

Tabla 19. Revistas localizadas en el JCRSS

Aquí, los países editores son México, Brasil, Chile y Colombia con un título por cada país.

\section{Conclusiones}

De todo lo anterior se puede concluir que efectivamente las revistas latinoamericanas en formato electrónico no son bien aceptadas todavía como publicaciones de calidad en las bases de datos internaciones.

También se puede concluir que los países con mayor crecimiento económico, científico y tecnológico son los que tienen una mayor presencia en estas bases de datos.

En las bases de referencias pertenecientes a las ciencias físico-matemáticas e ingenierías, la inclusión de revistas latinoamericanas es casi nula. $Y$ eso a pesar de que países como Brasil y México tienen una producción muy grande en este tema, que esta documentada en revistas de la especialidad internacionales.

Por su parte las áreas de Ciencias Sociales y Humanidades no son explotadas como debieran, ya que su presencia y factor de impacto es muy simbólico, apenas perceptible. Los países que representa la región son Brasil y México.

Otro de los problemas que se observaron son la cantidad de títulos que se han retirado de las bases de datos. Se aprecia que las revistas en formato solo electrónico no son bienvenidas en las bases de datos internacionales.

Por ultimo, las publicaciones latinoamericanas en formato electrónico cuentan con factores de impacto importantes. Sin embargo, al igual que ocurrre con las impresas, predominan las de las áreas de Ciencias y Tecnología, ya que localizamos 21 títulos de estas áreas y solo cuatro de Ciencias Sociales. Ello indicaría que este tipo de publicaciones no tienen una visibilidad amplia y que tienen una difusión tan solo regional. 


\section{Referencias}

Alonso Gamboa, José Octavio. (2003). Selección de revistas latinoamericanas en bases de datos: criterios utilizados en CLASE y PERIODICA. // Biblioteca Universitaria 6:1 (2003) 10-21.

Centro de Información Científica y Humanística (1994) http://dgedi.estadistica.unam.mx/memorias94/ClCH.htm (mayo 12, 2008).

Citas Latinoamericanas en Ciencias Sociales y Humanidades: CLASE. (2008). México: UNAM, Dirección General de Bibliotecas. http://dgb.unam.mx/clase.html (junio 10, 2008)

UNAM. Dirección de Estudios Administrativos (1977). Informe 1976. México. UNAM. p. 623.

UNAM. Dirección General de Bibliotecas (2004). http://dgb.unam.mx (Mayo 26, 2008).

Hela (2008). México : UNAM, Dirección General de Bibliotecas. http://dgb.unam.mx/hela.html (junio 10, 2008).

Índice de Revistas Latinoamericanas en Ciencias: PERIÓDICA. (2008). http://dgb.unam.mx/periodica.html (Junio 10, 2008).

Luna Castellanos, Rodolfo. (2004) Catálogo HELA,. // Exlibris 1:1 (2004) 4 .

Tapia Arroyo, Anabell (2008). La base de datos HELA: una experiencia de migración. México: UNAM, 2008. 